teienstatut und Parteienfinanzierung auf die Entwicklung der europäischen Parteien. Die folgenden sechs Beiträge von Claudia Ehmke, Janosch Steuwer und Siebo M. H. Janssen, Frank Schuster, Yvonne Nasshoven, Jürgen Mittag und Claudia Hülsken sowie Martin Schirdewan nehmen schließlich sehr versiert die "Professionalisierungsphase“ unter die Lupe. Obwohl die einzelnen Texte profunde Einblicke in die jüngere Parteienentwicklung liefern, wäre dem Leser noch besser gedient, wenn sich die Autoren - um einer leichteren Vergleichbarkeit willen - über eine einheitliche Struktur oder eine Einordnung ihres jeweiligen Gegenstandes entlang jener als „stilbildend“ genannten Kriterien von Kontakt, Kooperation und Interaktion Oskar Niedermayers verständigt hätten.

Den Abschluss bilden zwei weitere Texte, wobei derjenige von Axel Schäfer deutlich zu kurz geraten ist, um wirklich angemessen die Frage nach den Zukunftsaufgaben von Parteien zu beantworten. Erheblich besser gelingt es hingegen Jürgen Mittag und Eva Bräth, Perspektiven und Entwicklungslinien herauszuarbeiten. Insgesamt fällt deren Bilanz - durchaus folgerichtig - ernüchternd aus, weil sich die Parteien eben noch nicht zu jenem wichtigen Legitimationsanker entwickelt haben, den diese vielfach in den Nationalstaaten darstellen. An diesem Punkt schließt sich auch der Kreis zur Monographie Til Hubers, der bemerkt, dass „innerparteiliche und institutionelle Gegebenheiten eine demokratiefördernde Wirkung" verhindern (S. 109). Der Sammelband wird schließlich noch durch einen dokumentarischen Teil abgerundet, der sehr übersichtlich die zentralen Wendepunkte europäischer Parteiengeschichte zeigt.

Wer sich einen schnellen und untadeligen Überblick über die jüngere Entwicklung der SPE und ihre Funktionen verschaffen will, sollte sich getrost des Bandes von Til Huber bedienen; wer hingegen eine tiefgründigere Analyse europäischer Parteien insgesamt sucht, die zudem mit einer historischen Nachzeichnung ihres Werdens und Wachsens versehen ist, sollte den Sammelband von Jürgen Mittag zur Hand nehmen. Zur Beschreibung und Analyse der europäischen Parteienlandschaft leisten beide Publikationen auf ihre Weise einen wichtigen Beitrag.

Stephan Dreischer

\title{
Parteien in Osteuropa: aktuelle Bestandsaufnahmen
}

Bos, Ellen und Dieter Segert (Hrsg.): Osteuropäische Parteien als Trendsetter? Parteien und Parteiensysteme nach dem Ende des Übergangsjahrzehnts, Verlag Barbara Budrich, Opladen I Farmington Hills 2008, 352 Seiten, € 33,-.

Thieme, Tom: Hammer, Sichel, Hakenkreuz. Parteipolitischer Extremismus in Osteuropa: Entstehungsbedingungen und Erscheinungsformen, Nomos Verlagsgesellschaft, Baden-Baden 2007, 299 Seiten, € 39,-.

Mit dem von Ellen Bos und Dieter Segert herausgegebenen Sammelband erscheint nach längerer Zeit wieder ein aktueller Titel, der sich mit den Entwicklungen der Parteiensysteme Osteuropas auseinandersetzt. Gab es zu Beginn des Transformationsprozesses noch recht häufig Neuerscheinungen, die den Stand der Dinge dokumentierten, musste sich der 
interessierte Leser zumindest im deutschsprachigen Raum längere Zeit mit Einzelstudien zu bestimmten Ländern oder mit kürzeren Beiträgen, etwa nach Wahlen, in den entsprechenden Fachzeitschriften begnügen. Aber gerade aufgrund der weiterhin hohen Fluidität in Osteuropas Parteiensystemen ist es nach wie vor notwendig, diese Bestandsaufnahmen regelmäßig zu aktualisieren. Tom Thieme kommt mit seiner Dissertation sogar das Verdienst zu, ein bisher kaum bearbeitetes Feld zu erschließen. Wenngleich mittlerweile auch zur Extremismusforschung in Osteuropa einige Werke existieren, so muss doch konstatiert werden, dass die Forschung im Vergleich zu Westeuropa, wo die Zahl der wissenschaftlichen Veröffentlichungen zur extremen Rechten gerade in den letzten Jahren kaum noch zu überblicken ist und auch zur extremen Linken inzwischen eine Reihe von Studien vorliegt, noch in den Kinderschuhen steckt.

Der Sammelband von Ellen Bos und Dieter Segert überzeugt nicht nur in der Aktualität, sondern bietet auch eine große Bandbreite von untersuchten Ländern an. Neben den besser bekannten Fällen werden auch das Baltikum (von Axel Reetz) und der Kaukasus (von Margarete Klein) untersucht. Lediglich zur Region Ex-Jugoslawiens, aus der nur das von Antje Helmerich behandelte Parteiensystem Kroatiens ausgewählt wurde, hätte man sich zumindest einen Beitrag gewünscht, in dem ähnlich wie bei Baltikum oder Kaukasus ein Überblick zu mehreren Ländern gegeben wird. Dies wäre unter dem Aspekt der ethnischen Spannungen, die sich mit Ausnahme von Slowenien auch nach wie vor deutlich auf das Parteiensystem auswirken, wohl auch machbar gewesen.

Von dieser Einschränkung abgesehen liefert Teil II des Buches mit seinen Analysen der Parteiensysteme in Polen, Tschechien, der Slowakei und Ungarn, in Bulgarien, Rumänien und Kroatien sowie in den postsowjetischen Normalfällen Russland und der Ukraine und postsowjetischen Ausnahmen im Baltikum und dem Kaukasus eine gelungene aktuelle Übersicht. Lediglich die Einteilung in postsowjetische Normalfälle und Ausnahmen wird nicht näher begründet und erscheint auch dadurch fraglich, dass der Kaukasus mit seinen „Parteien der Macht“ ebenso wie Russland eher einen Normalfall darstellt. Dagegen steht das ukrainische Parteiensystem, das nach der „Orangenen Revolution“ zwar einen Schritt in Richtung Demokratisierung getan hat, aber nach wie vor stark von Oligarchen geprägt ist, in der Einstufung eher zwischen den Normalfällen und dem postsowjetischen Ausnahmefall, dem Baltikum, mit seinen zwar sehr instabilen, aber gegenüber den GUS-Staaten deutlich demokratischeren Parteiensystemen.

Der wesentliche Schwachpunkt des Sammelbandes liegt darin, dass die durch den Titel suggerierte Leitfrage, ob es sich bei den osteuropäischen Parteiensystemen um Trendsetter für Probleme handelt, die künftig auch in den etablierten Parteiensystemen Westeuropas auftauchen, sich nicht wie ein roter Faden durch das Buch zieht. So wird sie im mittleren Teil der Länderanalysen fast gar nicht aufgegriffen, sondern lediglich in Teil I (Theoretischer Rahmen) und Teil III (Erträge des Vergleichs) ernsthaft behandelt.

Nachdem Dieter Segert zu Beginn grundsätzliche Themen, die untersucht werden sollen, skizziert, folgen zwei Beiträge mit unterschiedlicher Qualität. So verliert sich Guido Tiemann in einer Unterscheidung zwischen Cleavages und Legacies und verpasst dabei völlig eine für die Leitfrage interessante Brücke zwischen den in Osteuropa noch schwach ausgeprägten und den in Westeuropa abnehmenden Parteibindungen durch Cleavages zu schlagen. Wesentlich gelungener ist hier der Beitrag von Jürgen Dieringer und Jane Óispuu über die Beeinflussung der Politik des Europäischen Parlaments durch die neuen Mitgliedsparteien. 
In Teil III analysieren Dorothée de Néve die mögliche Krise von Parteien und Susanne Fröhlich-Steffen die Rolle von Populisten. Hier wird nicht nur ein Vergleich zu Westeuropa gezogen, sondern auch zwei sehr aktuelle gesamteuropäische Problemfelder für Parteienforscher behandelt, wenngleich de Néve methodisch teils etwas schwach argumentiert (zum Beispiel Korruptionseinstufung lediglich anhand von Umfragen und nicht durch belegbare Daten). Am Ende des Buches schließen Ellen Bos und Dieter Segert die zu Beginn geöffnete Klammer zur Leitfrage: Sie kommen zu dem Ergebnis, dass sich die Parteiensysteme Osteuropas besonders gut für die Analyse von Problemen eignen, die auch im Westen zunehmend an Bedeutung gewinnen, aber in Osteuropa stärker ausgeprägt sind. Als Beispiel nennen sie etwa den schwindenden Einfluss der Nationalstaaten in der Globalisierung und die damit verbundene ansteigende Parteien- und Demokratieverdrossenheit der Bürger. In ihrer Argumentation beschränken sich die Autoren aber auf die im Buch behandelten Fälle, obwohl sie von ganz Osteuropa sprechen.

Im Mittelpunkt der Dissertation von Tom Thieme steht die Analyse von extremen politischen Parteien in Polen, Tschechien, der Slowakei, Ungarn und Russland. Der Untersuchung von insgesamt 14 Parteien liegt das normative Extremismuskonzept zu Grunde. Zur Einordnung der einzelnen Parteien entwirft Thieme ein Modell, das aus sechs Kriterien besteht, jeweils drei für historische wie für aktuelle Faktoren. Diese sind für den Autor „Merkmale, die in ihrer idealtypischen Form zwar Gegensätze von Rechts- und Linksextremismus markieren, sich aufgrund der erläuterten historischen und transformationsbedingten Extremismusursachen in Osteuropa jedoch nicht ausschließen müssen“ (S. 232). Im Einzelnen handelt es sich um die ideologische Grundlage, die Bewertung der Vergangenheit, die Symbolik, antidemokratische Äußerungen, das angestrebte Gesellschaftsmodell und die außenpolitischen Ambitionen der Parteien. So kommt er zu neun idealtypischen Organisationsformen, innerhalb derer er weitere Differenzierungen vornimmt, jedoch bezweifelt, dass diese Einteilung der Heterogenität der untersuchten Gruppierungen gerecht wird.

Die untersuchten Parteien kann er aber nur in vier der neun Kategorien einordnen. Zum eindeutig rechtsextremen Typ 1 zählt Thieme lediglich drei Parteien: die russischen RNE und Pamjat sowie die ungarische MIÉP; dem Typ 2 mit „rechts-links-durchmischter historischer Ideologie, aber eindeutig rechtsextremer Programmatik“ (S. 233) kann er hingegen keine Partei zuordnen; auch ist keine für ihn „undurchmischt linksextrem“ und keine dem „fast reinen Linksextremismus“ zuzurechnen. Die Mehrzahl der Parteien lässt sich für Thieme weder als nationalistisch noch als kommunistisch klassifizieren, sondern stellt Mischformen dar. Die polnische LPR oder die tschechischen Republikaner beispielsweise gelten als Typ 3 historisch eindeutig nationalistisch und aktuell rechts- wie linksextrem gemischt. Thieme spricht daher von einer „Durchmischung“ links- und rechtsextremer Elemente und antidemokratischen Mischformen.

Er kommt zu dem Schluss, dass herkömmliche Rechts-Links-Unterscheidungen in Osteuropa häufig nicht eindeutig treffen. Für ihn finden sich bei zahlreichen der untersuchten Parteien vielmehr sowohl Punkte, die eindeutig für eine Zuordnung zur extremen Rechten sprechen, wie solche, die klar für eine Klassifizierung als extrem links sprechen. Auf der einen Seite existieren laut Thieme Parteien, die eher internationalistisch als nationalistisch ausgerichtet seien und sich positiv auf den vergangenen Realsozialismus beziehen würden, aber auch eine rassistische Komponente beinhalteten; auf der anderen Seite gebe es ebenso nationalistische Parteien, die sich historisch an rechte Autokratien anlehnten, aber aus Gründen der Stimmenmaximierung ihre aktuelle Politik mit sozialistisch-antikapitalisti- 
schen Elementen versehen würden. Daher schlägt er vor, im Fall Osteuropas in der Regel nicht von links- oder rechtsextremistischen Parteien, sondern schlicht von extremistischen Parteien zu sprechen.

Unabhängig von diesem Ergebnis und der sich hier aufdrängenden, grundsätzlichen Frage der Einschätzung des Extremismuskonzepts lässt sich die Bewertung Thiemes im Detail nicht immer nachvollziehen. Zum Beispiel spricht er bereits bei der Erfüllung eines Kriteriums für Linksextremismus auf der Ebene der aktuellen Konflikte durch eine Partei von einer „zugleich rechts- und linksextremen Demokratiebedrohung“ und bezeichnet sie als „rechtslinks durchmischte extremistische Vereinigung“ (S. 235). Wenn er etwa angesichts der Tatsache, dass für manche der untersuchten Parteien im Kampf gegen Kriminalität der Zweck die Mittel heiligt und für öffentliche Sicherheit rechtsstaatliche Prinzipien wie das Primat der Unschuldsvermutung sowie der Schutz der Privatsphäre zu vernachlässigen seien und mit Hilfe eines staatlichen Sicherheitsapparates durchgesetzt werden sollen, diese „Forderungen nach Unterordnung des Einzelnen unter den Absolutheitsanspruch des staatlichen Kollektivs“ als „per se linksextreme Ziele“ (S. 235) bezeichnet, so löst dies Verwunderung aus, denn gerade der Ruf nach law-and-order gilt doch als Kernelement extrem rechter Politik.

Wer sich über aktuelle Entwicklungen in osteuropäischen Parteiensystemen informieren möchte oder einen informativen Einblick in ein wenig bearbeitetes Forschungsfeld gewinnen möchte, dem seien die Bücher von Bos und Segert sowie Thieme trotz der genannten Kritikpunkte zu empfehlen.

Jan Schedler und Karsten Schmitz

\section{Der Wehrbeauftragte des Deutschen Bundestages: nicht angemessen beurteilt}

Rosenow, Dörthe: Der Wehrbeauftragte im Transformationsprozess. Vom Kontrolleur zum Ombudsmann (Studien der Hessischen Stiftung Friedens- und Konfliktforschung, Band 3), Nomos Verlagsgesellschaft, Baden-Baden 2008, 162 Seiten, € 24,-.

Mit Dörthe Rosenows Buch, entstanden im Rahmen eines von der Hessischen Stiftung Friedens- und Konfliktforschung durchgeführten Forschungsprojekts, über das „Bild vom demokratischen Soldaten“, wird erstmals wieder seit längerer Zeit eine politikwissenschaftliche Abhandlung über das Amt des Wehrbeauftragten (WB) des Deutschen Bundestages vorgelegt. Die Studie gliedert sich in insgesamt sechs Kapitel. Zunächst wird ein knapper Abriss über das Amt des WB, seine Geschichte, Struktur und Aufgaben vorangestellt. Daran schließen sich Ausführungen zu den geänderten Rahmenbedingungen für die Bundeswehr und damit auch für das Amt des WB nach 1990 an, sowie eine theoretische Einbettung der Thematik, in deren Mittelpunkt die Hypothese der „Betriebsblindheit“ des WB steht. Der Hauptteil der Arbeit stützt sich auf die Auswertung der Jahresberichte der WB zwischen 1990 und 2005 sowie auf Interviews mit (ehemaligen) WB und auf Gespräche aus dem Arbeitsumfeld dieser Institution. Zum Abschluss werden die eingangs gestellten Hypothesen überprüft. 